Arq. Bras. Med. Vet. Zootec., v.68, n.3, p.611-619, 2016

\title{
Avaliação e comparação entre métodos de mensuração de pressão arterial sistólica em gatos hígidos conscientes
} [Evaluation and comparison between methods of measurement of systolic
blood pressure in healthy conscious cats]

\author{
T.M. Anjos ${ }^{1}$, J.C.C. Veado $^{1}$, R.B. Araújo ${ }^{1}$, F.O.P Leme $^{1}$, S.A. Diniz ${ }^{1}$, M.C.N. Castro ${ }^{2}$, F.V.A Costa ${ }^{3}$, \\ C.A.P. Tavares ${ }^{4}$, L.M. David ${ }^{4}$, K.X. Quites $^{4}$, M.Q. Maia ${ }^{4}$ \\ ${ }^{1}$ Escola de Veterinária - Universidade Federal de Minas Gerais - UFMG - Belo Horizonte, MG \\ ${ }^{2}$ Faculdade de Veterinária - Universidade Federal Fluminense - UFF - Niterói, RJ \\ ${ }^{3}$ Faculdade de Veterinária - Universidade Federal do Rio Grande do Sul - UFRGS - Porto Alegre, RS \\ ${ }^{4}$ Médico veterinário autônomo
}

\section{RESUMO}

Após validação de dois diferentes métodos de mensuração de pressão arterial sistólica (PAS) não invasivos ou indiretos (oscilométrico e Doppler vascular) com o invasivo ou direto (cateterização arterial - padrão ouro) em 12 gatos hígidos anestesiados de mesma faixa etária, os métodos não invasivos foram avaliados e comparados em 24 gatos hígidos e conscientes, divididos em quatro grupos de idade. Em cada animal, procedeu-se à mensuração da pressão pelos métodos Doppler e oscilométrico. $\mathrm{O}$ método oscilométrico foi o primeiro a ser realizado, e, logo em seguida, o método Doppler. Os valores considerados foram obtidos das médias de sete medidas consecutivas para cada método, com intervalo de 30 segundos entre elas. A primeira medida e as medidas discrepantes (variação $>20 \%$ PAS), obtidas com sinais óbvios de estresse e/ou com movimentação do animal, foram descartadas. Os métodos de mensuração oscilométrico e Doppler, embora tenham apresentado médias estatisticamente diferentes em todos os grupos, quando comparados em cada grupo, apresentaram uma correlação significativa, alta e positiva. Ou seja, sempre que for obtido um valor elevado por um método, o mesmo fato ocorrerá com o outro método e vice-versa. Clinicamente, a diferença observada não é relevante, uma vez que a diferença média dos valores obtida pelos dois métodos, em cada grupo, foi menor que $1,1 \%$.

Palavras-chave: felino, Doppler, oscilométrico, pressão arterial

\begin{abstract}
After two different validation systolic blood pressure measurement methods (PAS), not invasive or indirect (oscillometric and doppler vascular) with invasive or direct (arterial catheterization - Gold Standard) in 12 anesthetized healthy cats of the same age group, not invasive methods were evaluated and compared in 24 healthy conscious cats, divided into four age groups. In each animal, we proceeded to measure the pressure by Doppler and oscillometric methods. The oscillometric method was the first to be performed, and, shortly thereafter, the doppler method. The values considered were obtained from averages from seven consecutive measurements for each method with an interval of thirty seconds between them. The first measurement and disparate measurements (range $>20 \%$ PAS) obtained with obvious signs of stress and / or animal movement were discarded. Methods of oscillometric and doppler measurement, although statistically different averages were shown in all groups when compared, each group showed a significant high positive correlation, meaning that when a high value is obtained by a method, the same occurs with the other method, and vice versa. Clinically, the observed difference is not relevant since the mean difference values obtained by the two methods, in each group was lower than $1.1 \%$.
\end{abstract}

Keywords: feline, doppler, oscillometric, blood pressure

Recebido em 5 de outubro de 2015

Aceito em 8 de janeiro de 2016

E-mail: tathimouraoanjos@hotmail.com 


\section{INTRODUÇÃO}

Embora já tenham seu uso consagrado, os métodos indiretos de mensuração de pressão (não invasiva) ainda deixam dúvidas quanto à sua acurácia e praticidade na rotina clínica (Acierno et al., 2010). Faz-se necessário comparar e estabelecer métodos e aparelhos confiáveis que verdadeiramente auxiliem na mensuração fidedigna da PA de gatos e, consequentemente, no diagnóstico seguro da hipotensão e especialmente da hipertensão, doença esta de grande importância na clínica felina (Anjos, 2012).

A PA pode ser avaliada tanto por métodos indiretos quanto por método direto. $\mathrm{O}$ método direto ou invasivo oferece vantagens como monitoramento contínuo e preciso da PA, mesmo em condições de hipotensão grave, como em condições de hipertensão, sendo a mensuração intra-arterial considerada o padrão ouro (Brown et al., 2007). Em gatos, a cateterização intraarterial pode ser realizada por meio de dissecção de uma artéria ou de forma percutânea, sendo mais facilmente realizada na artéria femoral (Anjos et al., 2014), embora a artéria metatársica dorsal também possa ser utilizada (Trim, 1994). Raramente a técnica invasiva é utilizada na rotina devido às dificuldades e aos riscos, que incluem sedação e/ou anestesia (Henik, Dolson e Wenholz, 2005; Acierno et al., 2010), fatores que, além de pouco práticos, podem reduzir artificialmente a PA. Essa técnica é de difícil execução em animais de peso abaixo de $10 \mathrm{~kg}$, sendo desaconselhado seu uso em gatos (Stepien e Elliott in Elliott e Grauer, 2007). Complicações comuns decorrentes da cateterização são isquemia, formação de trombos, além de embolia. Por tais motivos, é mais utilizada em pesquisas acadêmico-científicas e unidades de terapia intensiva (Anjos et al., 2014).

Os métodos indiretos ou não invasivos, como o Doppler e o oscilométrico, são preferivelmente empregados em situações clínicas, devido à maior praticidade de uso e à possibilidade de serem repetidos em pequenos intervalos de tempo (Podell, 1992). Entretanto, são menos precisos em condições de hipotensão, vasoconstrição ou ainda quando ocorre movimentação excessiva do animal, podendo ser subestimado o verdadeiro valor da PA (Podell,
1992; Henik, Dolson e Wenholz, 2005; Anjos et al., 2014).

Ao contrário da medicina humana, na medicina veterinária não existem normativas bem definidas de validação de técnicas indiretas de mensuração de PA. Dessa forma, os equipamentos destinados à mensuração devem ser avaliados de acordo com as normas de validação exigidas por entidades internacionais, como a Association for Advancement of Medical Instrumentation (AAMI). A melhor forma de se avaliar um método indireto é compará-lo, de modo pareado, ao método invasivo (Bland e Altman, 1986). Ele deve apresentar uma diferença menor ou igual a cinco $\mathrm{mmHg}$, com desvio- padrão de, no máximo, $8 \mathrm{mmHg}$ (Association for the Advancement of Medical Instrumentation, 2003). Entretanto, o ANSI (Instituto Americano Nacional de Padrões, 2002) reconhece a recomendação publicada na normativa de 2001 do CEN (Comitê Europeu de Organização), que valida o método indireto se a diferença da pressão arterial diastólica (PAD) comparada ao método invasivo não for maior que $8 \mathrm{mmHg}$ e/ou se a PAS não for maior que $12 \mathrm{mmHg}$. De acordo com Brown et al. (2007), nenhum aparelho de mensuração indireta de PA ainda havia sido validado em medicina veterinária pelos critérios ora mencionados, até que Anjos e colaboradores (2014) validaram os métodos Doppler e oscilométrico para a espécie felina segundo esses critérios.

Objetivou-se com este estudo correlacionar os valores médios de PAS obtidos pelos aparelhos Doppler e oscilométrico, em gatos hígidos e conscientes, de diferentes faixas etárias, após validação prévia (Anjos et al., 2014) desses aparelhos.

\section{MATERIAL E MÉTODOS}

Foram selecionados 24 gatos (Felis catus) hígidos, sendo 12 machos e 12 fêmeas, com idade compreendida entre sete meses e 20 anos de idade, devidamente vacinados e desparasitados, de raças variadas, castrados, de porte e peso semelhantes. Foram divididos em quatro grupos de idade, sendo seis gatos em cada grupo (três machos e três fêmeas). Os gatos foram divididos em grupos Filhote/Júnior, Adulto, Maduro e Sênior/Geriátrico. 


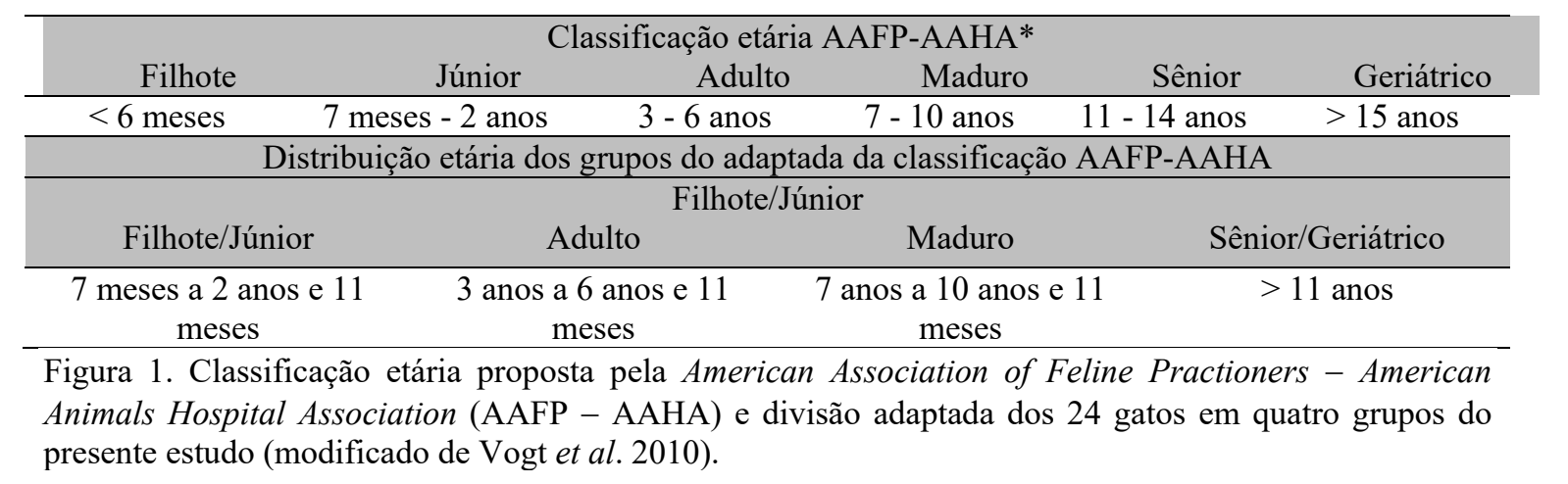

Os animais foram submetidos aos exames: físico completo, laboratoriais (sangue e urina) e de imagem (ultrassom abdominal e ecodopplercardiograma) para comprovação de higidez.

Inicialmente, o método oscilométrico foi realizado e, cinco minutos após o término, o mesmo procedimento foi repetido, entretanto empregando o método Doppler (membro torácico direito). Para ambos os métodos, utilizou-se o mesmo manguito, conforme recomendações do fabricante. Para obtenção de valores confiáveis de PA, todas as mensurações foram realizadas somente por um único examinador bem treinado, com experiência em mensuração de PA e familiaridade com os métodos e aparelhos estudados há mais de três anos. A primeira medida de PA obtida e as demais obtidas com valores extremos (variação > $20 \%$ PAS) foram excluídas. Foi seguido o protocolo (Brown et al., 2007) criado pelo Consenso "Diretrizes para a Identificação, Avaliação e Manejo de Hipertensão Arterial Sistêmica em Cães e Gatos", desenvolvido pelo Colégio Americano de Medicina Veterinária Interna e pela Sociedade Veterinária de Pressão Arterial.

Para a mensuração da PA, o gato foi posicionado no colo do tutor ou em posição confortável, e a técnica de mensuração foi realizada conforme descrito a seguir. Para mensuração pelo método oscilométrico (Petmap ${ }^{\circledR}$, Ramsey Medical, Inc, Estados Unidos), a escolha do manguito (CritterCuff size $-2.5 \mathrm{~cm} / 3.0 \mathrm{~cm} / 3.5 \mathrm{~cm} / 4.0 \mathrm{~cm}$, Ramsey Medical, Inc, Estados Unidos) era realizada conforme recomendações do fabricante, e o manguito colocado sempre no membro torácico direito sobre o terço distal da região radioulnar. $\mathrm{O}$ membro foi mantido posicionado ao nível do coração direito. O manguito, então, foi automaticamente inflado com um aperto contínuo da pera ao longo de um a três segundos a uma pressão suprassistólica $(40-50 \mathrm{mmHg})$ e, em seguida, desinflado automaticamente de modo progressivo. As oscilações da parede arterial emitidas pela pulsação sanguínea eram captadas pelo manguito e permitiam ao aparelho oscilométrico determinar a oscilação máxima e, consequentemente, calcular a PAM por meio do microprocessador contido dentro do aparelho. Logo, por cálculo aritmético, o aparelho determinava as PAS, PAD, além da frequência cardíaca. Para que nova mensuração pudesse ser realizada sem interferência, o manguito era totalmente desinflado, a fim de normalizar mais rapidamente a estase venosa. Segundo o fabricante do aparelho oscilométrico estudado, melhor precisão nas medidas de PA obtidas é alcançada utilizando-se o manguito de tamanho adequado, bem como observando-se o correto local de sua colocação, com as configurações adequadas para a espécie e a função de média que é oferecida pelo aparelho (após as mensurações seriadas, desliga-se o aparelho e liga-se novamente; na tela, aparecerá a leitura mais representativa daquela série de mensurações realizadas). Desse modo, mensurou-se a PA pelo método oscilométrico sempre no modo otimizado para a espécie felina e com o manguito colocado no membro torácico.

Após cinco minutos do término da técnica oscilométrica, o animal era mantido na mesma posição e com o mesmo manguito, para mensuração pelo Doppler vascular (Model $812^{\circledR}$, Parks Medical, Estados Unidos). O manguito era, então, desconectado do aparelho oscilométrico e conectado ao esfigmomanômetro aneroide (Esfigmomanômetro Gamma G5 ${ }^{\circledR}$, Heine, 
Alemanha). Álcool $70^{\circ}$ e gel de ecografia foram aplicados sobre a superfície do transdutor (Flat infant probe $8,2 \mathrm{MHz}$, Parks Medical Electronics, Inc, Estados Unidos) e sobre o pelo da região palmar metacarpal (artéria digital palmar comum) próximo ao coxim, onde o pulso era audível. Após obtenção dos sinais de pulso adequados, o manguito foi inflado até aproximadamente 40 a $50 \mathrm{mmHg}$ superior à pressão necessária para se obliterar o sinal de pulso audível e, logo após, a válvula do esfigmomanômetro foi aberta lentamente, sendo regulada manualmente para a liberação de ar na velocidade de 2 a $5 \mathrm{mmHg}$ por segundo, para que o manguito desinflasse lentamente. A PAS foi determinada no momento em que o sinal de pulso se tornou audível (claro e consistente).

Ambos os métodos foram realizados no mesmo dia e repetidos em três momentos distintos, em intervalo de sete dias, no curso de 21 dias; sempre nos mesmos horário e local (consultório climatizado com ar-condicionado e ambientado com difusor sintético de feromônio (Feliway Difusor, Feliway Spray, Ceva Sante Animale) sintético facial felino para dar mais segurança e tranquilidade aos gatos e minimizar o estresse). Para minimizar os efeitos causados pelo estresse ambiental (visual, olfativo e auditivo), o procedimento sempre foi realizado aos domingos, dia em que não havia funcionamento e animais ou pessoas próximas ao consultório. Ao chegar ao consultório, cada gato permaneceu cinco a 10 minutos sem qualquer tipo de manipulação, para que pudesse se acalmar e se adaptar ao ambiente, minimizando, dessa maneira, a "síndrome do jaleco branco". Todos os tutores estiveram presentes durante todos os momentos de mensuração de PA, com o intuito de também reduzir o estresse do animal, conforme recomendado pelo consenso de hipertensão (Brown et al., 2007).

O projeto foi aprovado pelo Comitê de Ética em Experimentação Animal (Cetea/UFMG), sob o protocolo de número 223/2011, com validade até 19/10/2016.

Os dados obtidos pelos métodos Doppler e oscilométrico de cada grupo foram analisados pela análise de variância (ANOVA), com teste pós-ANOVA, utilizando-se teste "t" de Student $(\mathrm{P}<0,05)$ para pareamento. Para comparação dos dois métodos entre os quatro grupos, foram utilizados o teste não paramétrico Kruskal-Wallis $(\mathrm{P}<0,05)$, bem como programas computacionais (softwares) BioStat 2009 Professional 5.8 .4 e Microsoft Excel.

\section{RESULTADOS E DISCUSSÃO}

Os métodos oscilométrico e Doppler, embora tenham apresentado médias estatisticamente diferentes em todos os grupos (Tab. 1 e Fig. 2), quando comparados em cada grupo, apresentaram uma correlação significativa, alta e positiva (Tab. 2), ou seja, sempre que for obtido um valor elevado por um método, o mesmo fato ocorrerá com o outro método e vice-versa. Clinicamente, a diferença observada não é relevante, uma vez que a diferença média dos valores obtidos pelos dois métodos, em cada grupo, foi menor que $1,1 \%$ (Tab. 1). Essa diferença não alteraria qualquer condição de pressão arterial, seja ela hipotensão, normotensão ou hipertensão, sem influenciar de modo importante na conduta clínica em relação ao paciente. Os resultados de PA apresentados neste experimento refletem a média das sete medidas consecutivas obtidas por cada método (42 medidas por gato, de cada um dos 24 gatos conscientes, totalizando 1008 medidas de PA), excluindo-se a primeira medida e as medidas discrepantes (variação maior que $20 \%$ da PAS), o que permite obter, em cada animal estudado, as sete medidas propostas na metodologia.

Os efeitos da idade nos valores da PA são bastante controversos (Jepson, 2011). Assim como nos trabalhos de Lawler et al. (1996) e Sparkes et al. (1999) e diferentemente de Sansom, Rogers e Wood (2004) e Carvalho (2009), não foi observado, neste estudo, aumento da PAS com o avançar da idade, quando se compara entre os diferentes grupos de idade. Apesar de não ter sido encontrada diferença significativa, quando se compararam os valores de PAS entre diferentes grupos de idade pelos dois métodos estudados (Tab. 1 e Fig. 2), percebe-se que as médias dos valores de PAS, tanto obtidas pelo oscilométrico $(142,57 \mathrm{mmHg})$ quanto pelo Doppler $(136,51 \mathrm{mmHg})$, são numericamente maiores no grupo Sênior/Geriátrico quando comparado aos demais grupos de idade (valores entre 128,02 e $133,02 \mathrm{mmHg}$ ). Esses valores, entretanto, são considerados normais para a espécie (Brown et al., 2007). Os valores encontrados sugerem que 
existe uma tendência à elevação da PA com o avançar da idade, e poder-se-ia supor que esses valores pudessem estar relacionados ao maior acometimento dos gatos idosos por afecções, como doença renal crônica, hipertireoidismo e/ou cardiomiopatia hipertrófica, doenças essas causadoras de hipertensão arterial sistêmica (HAS). Entretanto, no presente estudo, todos os exames de triagem descartaram quaisquer possibilidades de gatos portadores de doenças, subclínicas ou não, capazes de causar hipertensão. Dessa maneira, pôde-se evidenciar que todos os grupos de idade apresentaram valores de PA considerados normais para a espécie, mesmo os gatos mais velhos. Nos trabalhos (Mishina et al., 1998; Bodey e Sansom, 1998) descritos na literatura, nos quais se observou diferença significativa da PA nos gatos mais idosos, a elevação da PA nesses animais foi atribuída à ocorrência de doenças de base, subclínicas ou não, uma vez que nesses trabalhos foram estudadas populações heterogêneas compostas por gatos saudáveis, suspeitos e/ou portadores de doenças capazes de causar HAS. Além disso, em alguns trabalhos, não foi possível realizar exames de triagem para que os gatos portadores de alguma afecção pudessem ter sido descartados do estudo.

Mais importante do que o método de mensuração de pressão a ser utilizado é a consistência e atenção à técnica de mensuração. Deve-se ter em mente que nem sempre todas as medidas obtidas por determinado método de mensuração estarão dentro dos limites de exatidão de pressão. A PA se altera a cada batida do coração, mais do que cada vez que o animal respira, principalmente se o animal não estiver totalmente relaxado.

Tab. 1. Valores médios, diferenças ( $\mathrm{mmHg}$ e porcentagem) e desvios-padrão entre os diferentes métodos indiretos de mensuração de pressão arterial (oscilométrico e Doppler), segundo os diferentes grupos de gatos selecionados pela idade

\begin{tabular}{|c|c|c|c|c|c|}
\hline \multirow{2}{*}{\multicolumn{2}{|c|}{$\begin{array}{c}\text { Grupos } \\
\text { Pressão não invasiva }\end{array}$}} & \multirow[t]{2}{*}{$\begin{array}{c}\mathrm{N} \\
\text { (Gatos) }\end{array}$} & \multicolumn{2}{|c|}{$\begin{array}{c}\text { Métodos de mensuração } \\
\text { (Média e desvio-padrão - mmHg) }\end{array}$} & \multirow{2}{*}{$\begin{array}{l}\text { Diferença das médias } \\
\text { entre os dois métodos } \\
\quad(\mathrm{mmHg} \text { e } \%)\end{array}$} \\
\hline & & & Oscilométrico & Doppler & \\
\hline 1 & Filhote/Júnior & 6 & $133,02 \pm 7,07 \mathrm{a}, \mathrm{A}$ & 1 & Filhote/Junior \\
\hline 2 & Adulto & 6 & $129,92 \pm 11,43 \mathrm{a}, \mathrm{A}$ & $128,02 \pm 11,85 b, A$ & $1,90 \mathrm{mmHg} / 1,01 \%$ \\
\hline 3 & Maduro & 6 & $132,55 \pm 3,94 \mathrm{a}, \mathrm{A}$ & $129,28 \pm 3,05 \mathrm{~b}, \mathrm{~A}$ & $3,27 \mathrm{mmHg} / 1,02 \%$ \\
\hline 4 & Sênior/Geriátrico & 6 & $142,57 \pm 18,33 \mathrm{a}, \mathrm{A}$ & $136,51 \pm 18,61 \mathrm{~b}, \mathrm{~A}$ & $6,06 \mathrm{mmHg} / 1,04 \%$ \\
\hline
\end{tabular}

Letras minúsculas distintas na mesma linha demonstram diferença pelo teste de "t" de Student $(\mathrm{P}<0,05)$ e letras maiúsculas iguais na mesma linha e coluna demonstram igualdade pelo teste de kruskal-Wallis $(\mathrm{P}<0,05)$.

Tabela 2. Valores de coeficiente de correlação de Pearson entre os diferentes métodos indiretos de mensuração de pressão arterial (oscilométrico e Doppler), segundo os diferentes grupos de gatos selecionados pela idade

\begin{tabular}{|cccc|}
\hline \multicolumn{4}{|c|}{ Comparação entre métodos de mensuração - Oscilométrico x Doppler } \\
\hline Grupos PNI & Coeficiente de & Intervalo de confiança & $\mathrm{P}$ \\
& correlação de Pearson $\mathrm{r}$ & $95 \% \mathrm{r}$ & \\
$(\mathrm{x}, \mathrm{y})$ & $(\mathrm{x}, \mathrm{y})$ & \\
\hline
\end{tabular}

$\begin{array}{cccc}\text { Júnior } & 0,9392 & 0,54 \text { a } 0,99 & 0,0054 \\ \text { Adulto } & 0,9934 & 0,94 \text { a } 1,00 & 0,0001 \\ \text { Maduro } & 0,8628 & 0,17 \text { a } 0,98 & 0,0269 \\ \text { Sênior/Geriátrico } & 0,9927 & 0,93 \text { a } 1,00 & 0,0001\end{array}$




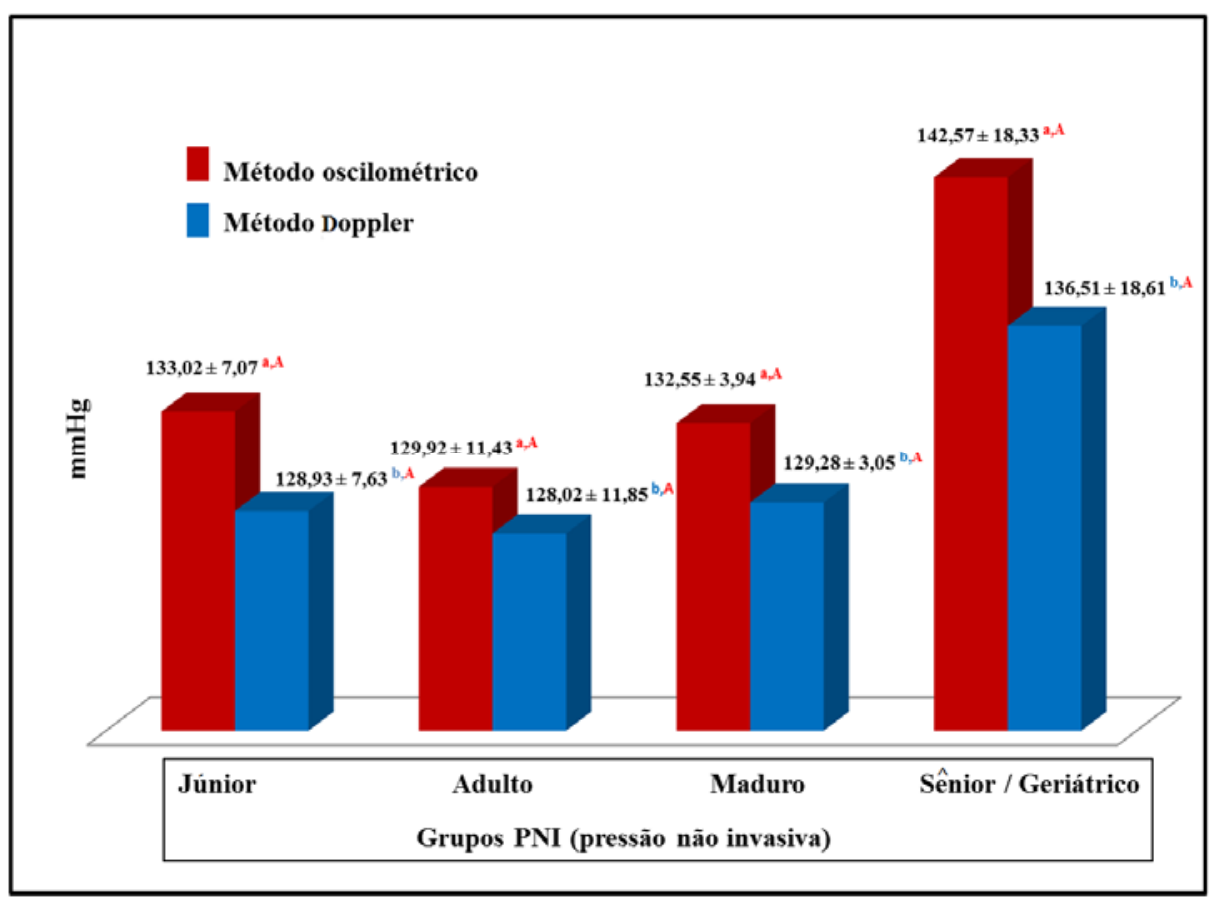

Figura 2. Representação gráfica dos valores médios e desvios-padrão obtidos mediante 21 mensurações (sete mensurações obtidas por cada método a cada sete dias, por três semanas consecutivas) de PA obtidas no primeiro momento pelo método oscilométrico e no segundo momento pelo método Doppler vascular (cinco minutos após o término da mensuração oscilométrica) em 24 gatos hígidos e conscientes, castrados, de porte e peso semelhantes, de diferentes raças, divididos em quatro diferentes grupos de idade (Filhote/Júnior, Adulto, Maduro e Sênior/Geriátrico) sendo seis animais por grupo, três machos e três fêmeas em cada.

A padronização do procedimento de mensuração de PA na clínica é fundamental, devendo ser esta sempre mensurada da mesma maneira em pacientes similares (por exemplo, anestesiados versus conscientes versus criticamente doentes). Idealmente, o operador deve ser bastante familiarizado com a técnica e treinado para executar o exame e registrar os resultados uniformemente. E o mais importante: a resposta fisiológica do felino ao estresse deve ser sempre observada atentamente. Ademais, condições ambientais devem ser criadas para que a mensuração da PA não crie um falso diagnóstico de hipertensão no felino ("síndrome do jaleco branco").

Como pode ser observado na Fig. 3, na maioria dos gatos estudados, não houve variações significativas nos três momentos distintos, demonstrando como é importante padronizar as técnicas e o ambiente de mensuração de PA para evitar obtenção de valores errôneos. Essas variações são esperadas pelas modificações fisiológicas contínuas de pressão. Entretanto, grandes variações podem interferir nos resultados e no diagnóstico do status pressórico do animal. Dessa maneira, além das mensurações seriadas em um dado momento, elas devem ser realizadas em momentos distintos para poder diagnosticar, de maneira fidedigna, a condição do paciente em relação à PA. Se o animal for submetido a uma única avaliação e, no dia, estiver estressado por qualquer razão, o médico veterinário terá um falso resultado de hipertensão arterial sistêmica. 


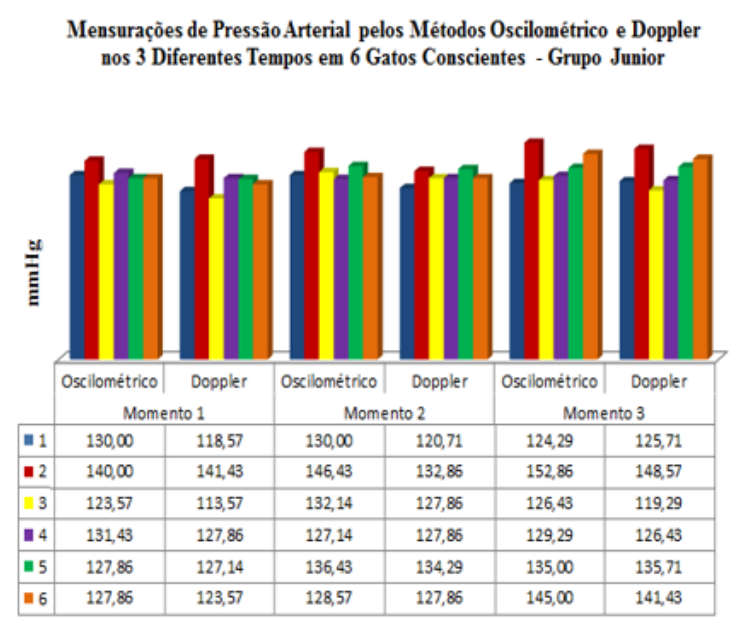

Mensurações de PressãoArterial pelos Métodos Oscilométrico e Doppler nos 3 Diferentes Tempos em 6 Gatos Conscientes - Grupo Maduro

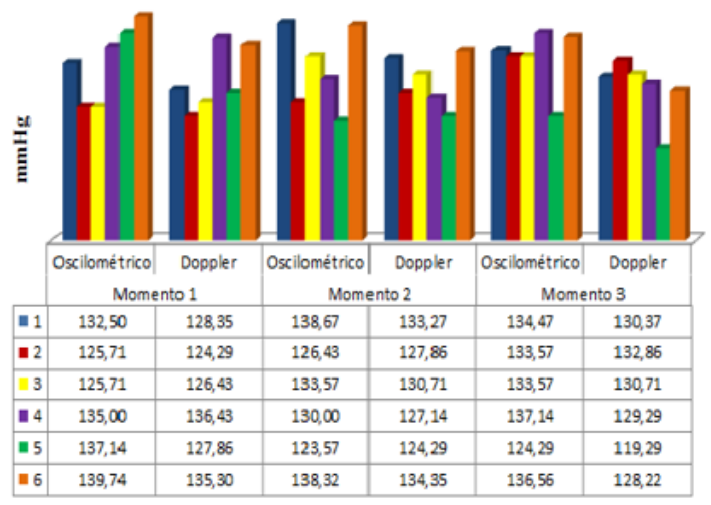

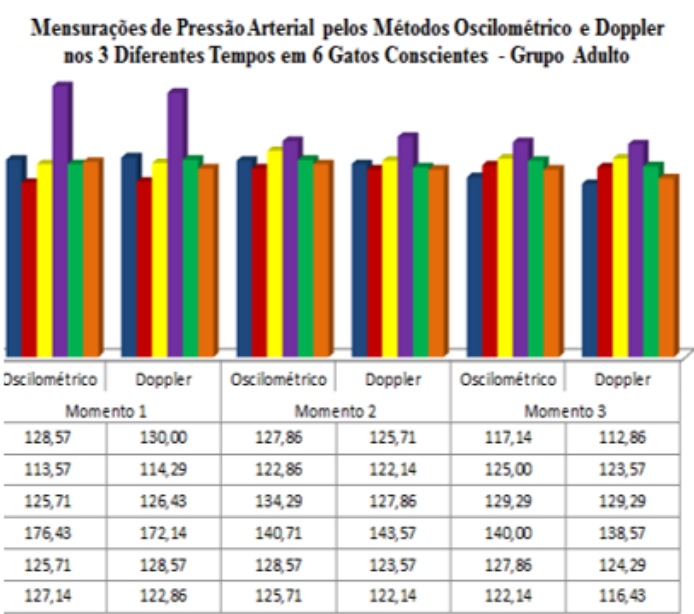

Mensurações de Pressão Arterial pelos Métodos Oscilométrico e Doppler s 3 Diferentes Tempos em 6 Gatos Conscientes - Grupo Senior / Geriátrico

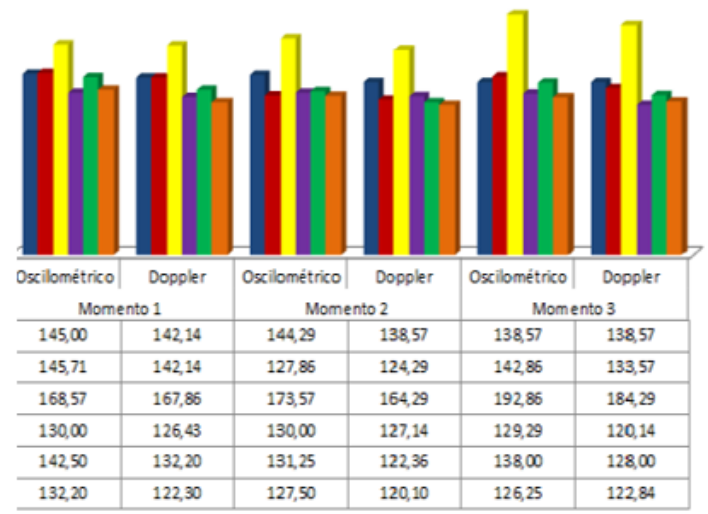

Figura 3. Valores médios $(\mathrm{mmHg})$ de PAS obtidos pelos métodos Doppler e oscilométrico, nos três momentos distintos de cada um dos gatos dos quatro grupos.

O comportamento fisiológico felino diante do estresse é extremamente relevante no momento de mensurar a PA. Ao se observar o comportamento do gato 4 do grupo Adulto e do gato 5 do grupo Maduro (fig. 3), por exemplo, demonstrou-se claramente que alguns animais, quando submetidos às mesmas condições ambientais e de manipulação e ao contato com as mesmas pessoas de maneira consecutiva e/ou frequente, podem se acostumar com essa situação e provar que os valores elevados de PA obtidos em um primeiro momento não são verdadeiramente o valor daquele animal, e sim valores causados pela "síndrome do jaleco branco". Se os valores de PA fossem considerados em apenas um único momento, este gato seria considerado um paciente hipertenso de acordo com a classificação de hipertensão segundo Brown et al. (2007), corroborando que as variações diárias de PA exigem um protocolo padrão de mensurações seriadas consecutivas (dias distintos) para oferecer a melhor estimativa de pressão arterial para gatos. $\mathrm{O}$ gato 6 do grupo Júnior demonstrou um comportamento oposto do gato 4 do grupo Adulto (Fig. 3), já que apresentou valores de PA, embora dentro da normalidade, bem mais elevados no último dia de mensuração, podendo ser devido ao estresse da manipulação constante e consecutiva, mesmo sob as mesmas condições. No último dia, esse animal estava bastante inquieto, difícil à manipulação e com alguns sinais visíveis de estresse, como taquipneia, e midríase. Alguns gatos podem não tolerar visitas frequentes ao 
consultório veterinário, tornando mais difícil a sua manipulação e qualquer tipo de intervenção médico-veterinária. Belew, Barlett e Brown (1999) demonstraram também que alguns gatos não se habituam a visitas periódicas. Já a gata 3 do grupo Sênior/Geriátrico (Fig. 3) também apresentou um comportamento parecido com o do gato 6 do grupo Júnior. Apesar de não ter apresentado nenhum indício de doença capaz de causar HAS, a gata 3 seria considerada hipertensa pelos valores de PA obtidos, entretanto era um animal extremamente estressado, mesmo na presença da tutora, em todos os dias em que foi manipulada. Tratava-se de uma paciente que não se acalmava mesmo após muitos minutos de aclimatação. Essa gata apresentava sinais visíveis de estresse, como vocalização, taquicardia, taquipneia, midríase e resposta exacerbada a qualquer tipo de manipulação, além dos valores elevados de PA. Esse animal poderia ter sido excluído do grupo devido ao seu comportamento e à dificuldade de manipulação dela, que, inclusive, interferiram nos resultados do grupo Sênior/Geriátrico, elevando as médias do grupo. Entretanto, optouse por mantê-la justamente para demonstrar que, mesmo sob condições consideradas ideais, gatos estressados dificultam o diagnóstico do seu estado pressórico e, consequentemente, a conduta do médico veterinário. Para gatos assim, talvez a melhor conduta pudesse ter sido mensurar a PA em seu próprio domicílio, no entanto esse não era o objetivo do estudo e, além disso, essa gata não permitia manipulação de maneira nenhuma, mesmo em seu domicílio. Alguns gatos são extremamente territorialistas e só se permitem ser manipulados fora de seu ambiente. Quimby, Smith e Lunn (2011) demonstraram que a avaliação clínica do paciente em domicílio não descarta alterações físiológicas causadas pelo estresse, já que foi observado que, em gatos menos tolerantes à sua manipulação dentro do seu território, os valores de PA obtidos no domicílio foram maiores que aqueles obtidos no consultório, demonstrando que, além da manipulação, como fonte de estresse, o territorialismo deve ser sempre levado em consideração.

Os efeitos da ansiedade e do paciente podem não ser previsíveis. Alguns animais apresentam um aumento dramático na PA, enquanto outros não, o que não significa que não estejam estressados por apresentarem mensurações com valores dentro da normalidade. Alguns ainda podem apresentar uma queda da PA (hiperatividade do sistema nervoso parassimpático) como resultado do processo de mensuração (Belew, Barlett e Brown, 1999; Anjos, 2012).

As mensurações de PA devem ser obtidas por meio de uma série de medidas para minimizar as alterações de PA (Brown et al., 2007), entretanto o processo de mensuração não deve se estender por um período muito longo (Lin et al., 2006), sob pena de o gato começar a ficar impaciente e ansioso, não permitindo a obtenção de valores fidedignos. De maneira contrária, alguns gatos só começam a apresentar valores confiáveis e consistentes após a obtenção de algumas medidas. O importante é que sejam excluídas a primeira medida e todas as demais com valores extremos (ou discrepantes) e incompatíveis com a média da série de medidas. Por essa razão, além das condições ambientais e das técnicas de mensuração bem padronizadas, é fundamental que o médico veterinário esteja bem atento ao comportamento do paciente, antes e principalmente durante a mensuração, para que possa ser capaz de perceber mudanças bruscas nos valores de PA devido às alterações fisiológicas geradas pelo estresse.

\section{CONCLUSÃO}

Não foi observado aumento da PAS com o avançar da idade quando comparada aos diferentes grupos de idade. Os métodos Doppler e oscilométrico diferem estatisticamente entre si (menor que $1,1 \%$ ), entretanto correlacionam-se de maneira alta e positiva, significando que, quando o valor de PAS for elevado em um método, esse também será pelo outro e viceversa. Apesar da diferença significativa entre os valores médios obtidos pelos métodos, em todos os grupos estudados, esses valores encontravamse dentro da faixa de normalidade, portanto não havendo relevância clínica. A coerência dos resultados das medidas realizadas, avaliada pela comparação entre os métodos estudados, está diretamente relacionada aos cuidados aplicados à metodologia de mensuração de pressão arterial, pelas técnicas Doppler e oscilométrica. 


\section{REFERÊNCIAS}

ACIERNO, M.J.; SEATON, D.; MITCHELL, M.A. et al. Agreement between directly measured blood pressure and pressures obtained with three veterinary-specific oscillometric units in cats. J. Am. Vet. Med. Assoc., v.237, p.402406, 2010.

ANJOS, T.M. Hipertensão arterial sistêmica em medicina felina. 2012. 50f. Trabalho de Conclusão de Curso (Pós-graduação lato sensu em Clínica Médica e Cirúrgica de Felinos) Universidade Paulista, Belo Horizonte, MG.

ANJOS, T.M.; VEADO, J.C.C.; CASTRO, M.C.N. et al. Avaliação e comparação entre métodos de mensuração de pressão arterial sistólica em gatos hígidos anestesiados. Arq. Bras. Med. Vet. Zootec., v.66, n.4, p.1151-1159, 2014.

BELEW, A.M.; BARLETT, T.; BROWN, S.A. Evaluation of the white-coat effect in cats. J. Vet. Intern. Med., v.13, p.134-142, 1999.

BLAND, J.M.; ALTMAN, D.G. Statistical methods for assessing agreement between two methods of clinical measurement. The Lancet, v.327, p.307-310, 1986.

BODEY, A.R.; SANSOM, J. Epidemiological study of blood pressure in domestic cats. J. Small Anim. Pract., v.39, p.567-573, 1998.

BROWN, S.; ATKINS, C.; BAGLEY, R. et al. Guidelines for the identification, evaluation, and management of systemic hypertension in dogs and cats. J. Vet. Intern. Med., v.21, p.542-558, 2007.

CARVALHO, V.L.A.B. Hipertensão arterial felina. 2009. 114f. Dissertação (Mestrado Integrado em Medicina Veterinária) - Faculdade de Medicina Veterinária, Universidade Técnica de Lisboa, Lisboa, Portugal.

HENIK, R.A; DOLSON, M.K.; WENHOLZ, B.S. How to obtain a blood pressure measurement. Clin. Tech. Small Anim. Pract., v.20, p.144-150, 2005.

JEPSON R.E. Feline Systemic Hypertension Classification and pathogenesis. J. Feline Med. Surg., v.13, p.25-34, 2011.

LAWLER, D.F.; KLETNER, D.G.; BINNS, S. et al. Age related differences in feline blood pressure (abstract). Vet. Clin. Nutr., v.3, p.29, 1996.
LIN C-H., YAN, C-J., LIEN, Y-H. et al. Systolic blood pressure of clinically normal and conscious cats determined by an indirect doppler method in a clinical setting. J. Vet. Med. Sci., v.68, p.827-832, 2006.

MANUAL electronic, or automated sphygmomanometers. 2003. Arlington: ASSOCIATION FOR THE ADVANCEMENT OF MEDICAL INSTRUMENTATION, 2003. $586 \mathrm{p}$.

MISHINA, M.; WATANABE, T.; FUJI, K. et al. Non-invasive blood pressure measurements in cats: clinical significance of hypertension associated with chronic renal failure. J. Vet. Med. Sci., v.60, p.805-808, 1998.

PODELL M. Use of blood pressure monitors. In: BONAGURA, J.D. Kirk's current veterinary therapy XI: small animal practice. Philadelphia, PA: WB Saunders. 1992. p.834-837.

QUIMBY, J.M.; SMITH, M.L.; LUNN, K.F. Evaluation of the effects of hospital visit stress on physiologic parameters in the cat. J. Feline Med. Surg., v.13, p.733-737, 2011.

SANSOM, J.; ROGERS, K.S., WOOD, J.L.N. Blood pressure assessment in healthy cats and cats with hypertensive retinopathy. Am. J. Vet. Res., v.65, p.245-252, 2004.

SPARKES, A.H.; CANEY, S.M.; KING, M.C. et al. Inter - and intraindividual variation in Doppler ultrasonic indirect blood pressure measurements in healthy cats. J. Vet. Int. Med., v.13, p.314-318, 1999.

STEPIEN, R.L.; ELLIOTT, J. Measurement of blood pressure. In: ELLIOTT, J.; GRAUER, G.F. BSAVA manual of canine and feline nephrology and urology. Quedgeley, Glouceste: British Small Animal Veterinary Association, 2007. p.178-191.

TRIM, C.M. Monitoring the anaesthetized cat. In: HALL, L.W.; TAYLOR, P.M. Anaesthesia of the cat. London: Baillière Tindal. 1994. p.194223.

VOGT A.H.; RODAN I.; BROWN M.; BROWN S. et al. AAFP - AAHA Feline Life Stage Guidelines. J. Feline Med. Surg., v.12, p.43-54, 2010. 